\title{
Cavernous Hemangioma of the Rib: A Case Report
}

\author{
Joon Young Park, ${ }^{1}$ Jin gyoon Park, ${ }^{1}$ and Seung Jin Lee ${ }^{1,}{ }^{*}$ \\ ${ }^{1}$ Department of Radiology, Chonnam National University Hospital, Gwangju, Korea \\ "Corresponding author: Seung Jin Lee, Department of Radiology, Chonnam National University Hospital, Gwangju, Korea. Tel: +82-622205146, Fax: +82-622264380, E-mail: \\ seungjinlee527@gmail.com
}

Received 2015 July 21; Revised 2016 April 06; Accepted 2016 May 08.

\begin{abstract}
Hemangioma of the rib is a rare benign vascular tumor. Herein, we report a 63-year-old man presenting with hemangioma of the left sixth rib. An osteolytic eccentric expansive mass with calcification and focal cortical disruption was detected on chest computed tomography (CT). The mass showed low 2-deoxy-2-[18F]-fluoro-D-glucose (18F-FDG) avidity on positron emission tomography. The patient underwent resection of the left sixth rib. Based on the histopathology findings, a diagnosis of cavernous hemangioma was made. Rib hemangiomas and malignant lesions appear similar on CT scans; therefore, most cases of rib hemangiomas reported in the literature were treated with rib resection. However, rib hemangiomas should be included in the differential diagnosis of an asymptomatic patient if an osteolytic expansive lesion containing sunburst calcifications with low 18F-FDG avidity is observed on a CT scan.
\end{abstract}

Keywords: Rib Resection, Hemangioma, Tumor

\section{Introduction}

Bone hemangiomas account for about $1 \%$ of all bone tumors and commonly involve the vertebrae and the skull (1). Hemangioma of the rib is uncommon and its diagnosis based on its 2-deoxy-2-[18F]-fluoro-D-glucose (18F-FDG) avidity on positron emission tomography (PET) scan is extremely rare. Herein, we report a 63-year-old man in whom a hemangioma of the left sixth rib was incidentally detected on a plain radiograph. For further investigation, computed tomography (CT) and 18F-FDG PET were performed.

\section{Case Presentation}

A 63-year-old man was admitted to our hospital for evaluation of an incidentally detected rib mass on a plain chest radiograph. The patient was taking antihypertensive medication and had an otherwise unremarkable medical history. The laboratory investigation values including serum tumor marker levels were within normal ranges.

An anterior-posterior view of a plain chest radiograph taken at another outpatient clinic revealed a moderately defined mass in the left upper hemithorax arising from the sixth rib (Figure 1). For further evaluation, CT scan (Somatom definition flash, Siemens healthcare, Forchheim, Germany) was performed. It demonstrated an osteolytic eccentric expansive mass with sunburst calcification and focal cortical disruption at the posterolateral arc of the left sixth rib, measuring $5.5 \times 2.5 \times 1.5 \mathrm{~cm}$ (Figure 2). Based on CT scan findings, differential diagnoses of malignant tumors, including chondrosarcoma, metastatic tumor, or multiple myeloma were considered. To distinguish between the differential diagnoses, a 18F-FDG PET/CT scan was obtained (GE, Discovery, Milwaukee, WI, USA), on which the lesion showed low 18F-FDG avidity (the maximum standardized uptake value [SUVmax] was 2.4). No other significant 18F-FDG uptake was observed on the PET/CT scan (Figure 3 ).

The patient underwent rib resection via posterolateral thoracotomy approach. A definite diagnosis of cavernous hemangioma was made based on histopathology examination results of the resected mass (Figure 4 ). The mass was composed of thin-walled blood vessels with dilated channels containing red blood cells and lined by a single layer of endothelial cells.

\section{Discussion}

Hemangioma is a benign neoplasm of blood vessels that can occur throughout the body. Histologically, there are four types of hemangiomas: cavernous, capillary, venous, and mixed type. Cavernous hemangiomas are the most common and account for up to $50 \%$ of all hemangiomas. They consist of dilated vessels lined by a single layer of endothelial cells surrounded by a fibrous stromal layer. Most cavernous hemangiomas involve the medullary and intracortical portion of the bone. Capillary 


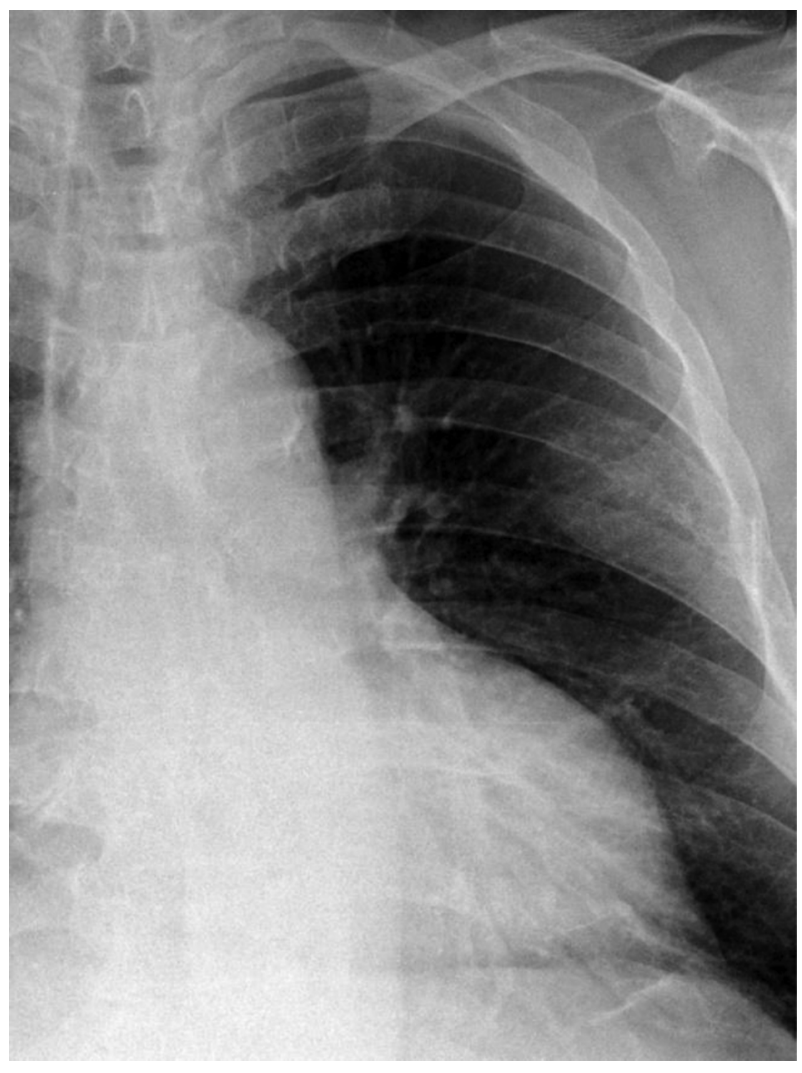

Figure 1. A 63-year-old man presenting with an incidentally detected rib mass on a plain chest radiograph diagnosed as hemangioma. Plain radiograph of the chest illustrates a moderately defined mass in the left upper hemithorax arising from the sixth rib.
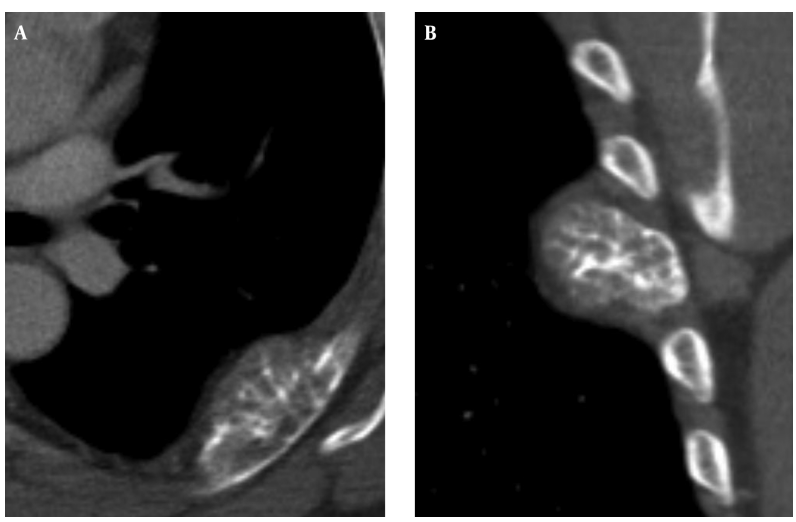

Figure 2. Axial contrast-enhanced chest CT image (A) and coronal reformation image (B) show an osteolytic eccentric expansive mass in the posterolateral part of the left sixth rib with sunburst calcification and focal cortical disruption.

hemangiomas account for $10 \%$ of all hemangioma cases and consist of multiple tortuous small vessels lined with epithelium, while venous and mixed type hemangiomas
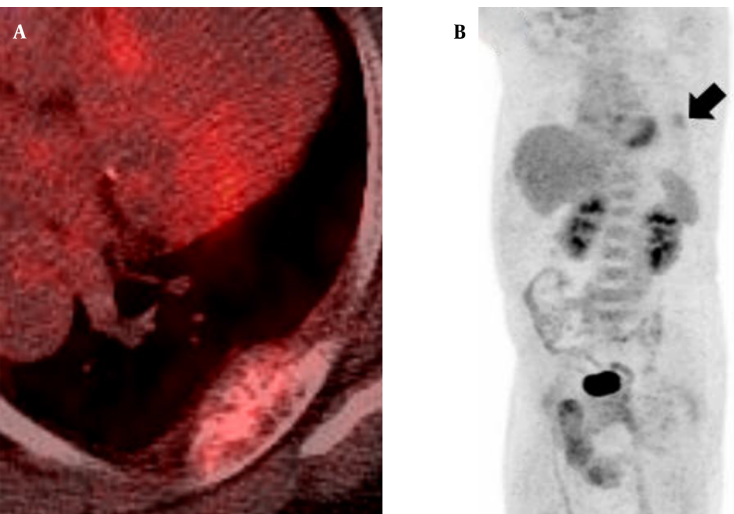

Figure 3. Axial 18F-FDG PET/CT fusion image (A) and MPI image (B) demonstrate nondiscrete focal 18F-FDG uptake (SUVmax 2.4) in the left sixth rib (arrow) without any other abnormal 18F-FDG uptake lesion.
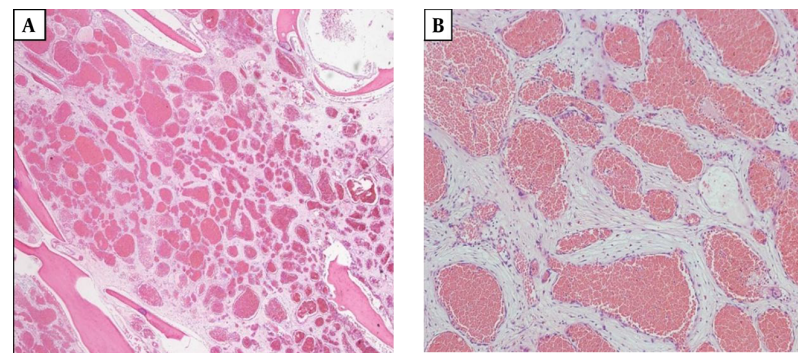

Figure 4. Microscopic findings of the resected mass. A, Normal bone tissues are interspersed within the mass (hematoxylin-eosin, original magnification $\times 20$ ). B, The mass consists of thin-walled blood vessels with single layer of endothelial-cell lining containing red blood cells (hematoxylin-eosin, original magnification $\times 100$ ).

are rare (2).

Most cases of bone hemangiomas develop in the vertebral body or the skull (1). Hemangioma of the rib is rare, both as a rib tumor and as a bone hemangioma (3). It is mostly detected incidentally as it is generally asymptomatic (4). However, about $50 \%$ of rib tumors are malignant, and it is difficult to distinguish a rib hemangioma from a malignant tumor such as a chondrosarcoma, metastatic tumor, or multiple myeloma (5). Bone hemangiomas can present with different features on scans obtained using varying imaging techniques. Hemangiomas in flat bones including the ribs can appear as aggressive tumors demonstrating cortical destruction with extraosseous mass formation and osteolytic lesions. As these imaging findings are typically observed in malignant lesions, the diagnosis of a rib hemangioma based on imaging findings alone might be challenging.

18F-FDG PET can detect the elevated glucose metabolism of cells, and is widely used for differentiation between benign and malignant neoplasms. Malignant le- 
sions tend to be 18F-FDG avid and benign lesions generally show lower 18F-FDG avidity (6). A benign lesion showing typical low FDG avidity includes osteochondroma, enchondroma, hemangioma, and intraosseous lipoma (7-12). Choi et al. reported that the mean SUVmax values in the benign rib lesions were $2.5 \pm 1.1$ (13). The SUVmax of our patient's lesion was 2.4, which implied that the lesion was more likely to be a benign tumor rather than a malignant one.

Rib hemangiomas and malignant bone lesions can appear the same on imaging scans; therefore, most of the patients with a rib hemangioma undergo rib resection. However, if CT scans show an osteolytic expansive lesion containing sunburst calcifications with low 18F-FDG avidity, a diagnosis of rib hemangioma should be considered.

\section{Footnotes}

Authors' Contributions: Joon Young Park carried out the final preparation of the manuscript. Jin gyoon Park contributed to the discussion in the conclusion, and critically reviewed the manuscript. Seung Jin Lee contributed to the final preparation of the manuscript. All authors contributed to the content and approved the final version of the manuscript.

Declaration of Interest: The authors report no declaration of interest.

Financial Disclosure: None declared.

Funding/Support: None declared.

\section{References}

1. Dorfman HD, Steiner GC, Jaffe HL. Vascular tumors of bone. Hum Pathol. 1971;2(3):349-76. [PubMed: 4940691].
2. Jain SK, Songra M, Malhotra A, Kapoor N, Malik R, Shrivastava A. Rib haemangioma: a rare differential for rib tumours. Indian J Surg. 2011;73(6):447-9. doi: 10.1007/s12262-011-0278-6. [PubMed: 23204706].

3. Okumura T, Asamura H, Kondo H, Matsuno Y, Tsuchiya R. Hemangioma of the rib: a case report. Jpn J Clin Oncol. 2000;30(8):354-7. [PubMed: 11059341].

4. Gourgiotis S, Piyis A, Panagiotopoulos N, Panayotopoulos P, Salemis NS. Cavernous hemangioma of the rib: a rare diagnosis. Case Rep Med. 2010;2010:254098. doi: 10.1155/2010/254098. [PubMed: 20585365].

5. Kuo YT, Lin MB, Sheu RS, Liu GC, Chai CY, Chou SH. Imaging diagnosis of cavernous hemangioma of the rib-one case report and review of the literature. Gaoxiong Yi Xue Ke Xue Za Zhi. 1994;10(8):469-73. [PubMed: 7799468].

6. Choi YY, Kim JY, Yang SO. PET/CT in benign and malignant musculoskeletal tumors and tumor-like conditions. Semin Musculoskelet Radiol. 2014;18(2):133-48. doi: 10.1055/s-0034-1371016. [PubMed: 24715446].

7. Costelloe CM, Chuang HH, Chasen BA, Pan T, Fox PS, Bassett RL, et al. Bone Windows for Distinguishing Malignant from Benign Primary Bone Tumors on FDG PET/CT. J Cancer. 2013;4(7):524-30. doi: 10.7150/jca.6259. [PubMed: 23983816].

8. Schulte M, Brecht-Krauss D, Heymer B, Guhlmann A, Hartwig E, Sarkar MR, et al. Grading of tumors and tumorlike lesions of bone: evaluation by FDG PET. J Nucl Med. 2000;41(10):1695-701. [PubMed: 11038000].

9. Aoki J, Watanabe H, Shinozaki T, Takagishi K, Ishijima H, Oya N, et al. FDG PET of primary benign and malignant bone tumors: standardized uptake value in 52 lesions. Radiology. 2001;219(3):774-7. doi: 10.1148/radiology.219.3.r01ma08774. [PubMed: 11376267].

10. Tian R, Su M, Tian Y, Li F, Li L, Kuang A, et al. Dual-time point PET/CT with F-18 FDG for the differentiation of malignant and benign bone lesions. Skeletal Radiol. 2009;38(5):451-8. doi:10.1007/s00256-008-06430. [PubMed: 19205695].

11. Shin DS, Shon OJ, Han DS, Choi JH, Chun KA, Cho IH. The clinical efficacy of (18)F-FDG-PET/CT in benign and malignant musculoskeletal tumors. Ann Nucl Med. 2008;22(7):603-9. doi:10.1007/s12149-008-01512. [PubMed: 18756363].

12. Costelloe CM, Chuang HH, Madewell JE. FDG PET/CT of primary bone tumors. AJR Am J Roentgenol. 2014;202(6):W521-31. doi: 10.2214/AJR.13.11833. [PubMed: 24848845].

13. Choi HS, Yoo Ie R, Park HL, Choi EK, Kim SH, Lee WH. Role of (1)(8)FFDG PET/CT in differentiation of a benign lesion and metastasis on the ribs of cancer patients. Clin Imaging. 2014;38(2):109-14. doi: 10.1016/j.clinimag.2013.11.011. [PubMed: 24361174]. 